\title{
Chapter 9 \\ Yeast at the Forefront of Research on Ageing and Age-Related Diseases
}

\author{
Belém Sampaio-Marques, William C. Burhans and Paula Ludovico
}

\begin{abstract}
Ageing is a complex and multifactorial process driven by genetic, environmental and stochastic factors that lead to the progressive decline of biological systems. Mechanisms of ageing have been extensively investigated in various model organisms and systems generating fundamental advances. Notably, studies on yeast ageing models have made numerous and relevant contributions to the progress in the field. Different longevity factors and pathways identified in yeast have then been shown to regulate molecular ageing in invertebrate and mammalian models. Currently the best candidates for anti-ageing drugs such as spermidine and resveratrol or anti-ageing interventions such as caloric restriction were first identified and explored in yeast. Yeasts have also been instrumental as models to study the cellular and molecular effects of proteins associated with age-related diseases such as Parkinson's, Huntington's or Alzheimer's diseases. In this chapter, a review of the advances on ageing and age-related diseases research in yeast models will be made. Particular focus will be placed on key longevity factors, ageing hallmarks and interventions that slow ageing, both yeast-specific and those that seem to be conserved in multicellular organisms. Their impact on the pathogenesis of age-related diseases will be also discussed.
\end{abstract}

Keywords Ageing $\cdot$ Nutrient-sensing pathways $\cdot$ Autophagy $\cdot$ Chronological life span $\cdot$ Replicative life span $\cdot$ Yeast $\cdot$ Proteostasis

\footnotetext{
B. Sampaio-Marques · P. Ludovico ( $₫)$

Life and Health Sciences Research Institute (ICVS), School of Medicine, University of Minho, 4710-057 Braga, Portugal

e-mail:pludovico@med.uminho.pt

B. Sampaio-Marques

e-mail: mbmarques@med.uminho.pt

B. Sampaio-Marques · P. Ludovico

ICVS/3B's - PT Government Associate Laboratory, Braga/Guimarães, Portugal

W. C. Burhans

Department of Molecular and Cellular Biology, Roswell Park Cancer Institute, Buffalo, NY

14263, USA

e-mail:wburhans@buffalo.edu
} 


\section{Abbreviations}

$\begin{array}{ll}\text { aSyn } & \text { Alpha-synuclein } \\ \text { AD } & \text { Alzheimer's disease } \\ \text { A } \beta & \text { Amyloid- } \beta \\ \text { AMPK } & \text { AMP-activated protein kinase } \\ \text { ATG } & \text { Autophagy gene } \\ \text { CLS } & \text { Chronological life span } \\ \text { CORE } & \text { Cross-organelle stress response } \\ \text { CR } & \text { Caloric restriction } \\ \text { DDR } & \text { DNA damage responses } \\ \text { ERCs } & \text { Extrachromosomal rDNA circles } \\ \text { HD } & \text { Huntington's disease } \\ \text { Htt } & \text { Huntingtin } \\ \text { IPOD } & \text { Insoluble protein deposit } \\ \text { INQ } & \text { Intranuclear quality control compartment } \\ \text { GTA } & \text { Genotoxin-induced targeted autophagy } \\ \text { JUNQ } & \text { Juxta nuclear quality control site } \\ \text { NQ } & \text { Non-quiescent } \\ \text { OXPHOS } & \text { Oxidative phosphorylation } \\ \text { PD } & \text { Parkinson's disease } \\ \text { PAS } & \text { Phagophore assembly site } \\ \text { PKA } & \text { Protein kinase A } \\ \text { Pho85 } & \text { Phosphate metabolism protein 85 } \\ \text { PolyQ } & \text { Polyglutamine } \\ \text { Q } & \text { Quiescent } \\ \text { ROS } & \text { Reactive oxygen species } \\ \text { RLS } & \text { Replicative life span } \\ \text { RNR } & \text { Ribonucleotide reductase } \\ \text { Snf1 } & \text { Sucrose non-fermenting protein 1 } \\ \text { TOR } & \text { Target of rapamycin } \\ \text { TORC1 } & \text { Target of rapamycin complex 1 } \\ \text { UPS } & \text { Ubiquitin proteasome system } \\ \text { VPS } & \text { Vacuolar protein sorting } \\ & \end{array}$

\subsection{Introduction}

Ageing is a complex and multifactorial process driven by genetic, environmental and stochastic factors that lead to the cumulative incorporation of imbalances at the genomic and proteomic level in a multidimensional process resulting in the progressive decline of biological systems and decreased cellular fitness over time. In spite the fact that eukaryotic species have their own set of age-related diseases, the hallmarks 
of cellular ageing are surprisingly conserved. These include primary events that trigger the progressively accumulation of cellular damage with ageing such as genomic instability, epigenetic alterations, loss of proteostasis, deregulated nutrient-sensing and mitochondrial dysfunction (reviewed in Lopez-Otin et al. (2013)). Due to the well-conserved hallmarks of cellular ageing, the budding yeast Saccharomyces cerevisiae has been widely used as a model of cellular and organismal ageing (Kaeberlein 2010; Longo et al. 2012; Sampaio-Marques et al. 2014a). The first study of yeast ageing, published more than 60 years ago, showed that yeasts have a finite replicative capacity (Mortimer and Johnston 1959). Mortimer and Johnston plotted their data and made the remarkable observation that the mortality curve for a yeast population resembles the mortality curves for many other organisms, including humans (Mortimer and Johnston 1959). Based on this observation, Replicative Life Span (RLS) was defined as the number of daughter cells produced by a single mother cell before dying. This definition underlies what has become a valuable model for studying ageing of mitotic cells. A second yeast model of ageing-the Chronological Life Span (CLS) model—was first proposed for budding yeast in 1980 (Muller et al. 1980). CLS is defined as the time that yeast cells can survive in a non-dividing state after exhaustion of the carbon source (Fabrizio and Longo 2003). Therefore, this single-celled organism provides a unique opportunity to study the ageing of both mitotic and post-mitotic cells (Kaeberlein et al. 2007). Not surprisingly, much of the advances on ageing research can be traced back to yeast that facilitates discovery of the evolutionarily conserved molecular and cellular mechanisms through which genetic and environmental interventions promote longevity. Yeasts were especially pivotal in the discovery of sirtuins and the TOR signalling pathway linking environmental nutrients to longevity. Different studies using these two yeast models of ageing have found that reducing glucose in the media can increase both RLS and CLS (Fabrizio and Longo 2003; Jiang et al. 2000). This paradigm linking environmental nutrients to longevity has been referred to as calorie restriction (CR) and is a major focus on ageing research.

This chapter intends to present some of the leading evidence and relevant advances on ageing and age-related diseases research in yeast models, with particular focus on longevity-promoting effects and proteostasis control.

\subsection{Genes and Pathways Modulating Yeast Ageing: Replicative Versus Chronological Life Span}

The two yeast models of ageing constitute important paradigms for the progressive accumulation of damage during ageing. While in the Replicative Life Span (RLS) damage is accumulated in mother cells due to the asymmetrical inheritance of damage, in the Chronological Life Span (CLS), the non-dividing cells cannot dilute the damage accumulated during ageing. Although both ageing models have different molecular and genetic determinants, they are not entirely independent. Indeed, there 
is evidence that chronological aged cells have reduced RLS once they re-enter into the cell cycle (Ashrafi et al. 1999; Murakami et al. 2012; Piper 2006). Furthermore, some longevity-promoting interventions extend both CLS and RLS, while defects in protein quality control contribute to decreased survival in both ageing models (reviewed in Sampaio-Marques et al. (2014a)). This evidence gives strong support to damage-based theories of ageing but also recognizes that some molecular and genetic factors play a key role in ageing.

The key finding that defines the RLS is the fact that individual cells have a finite number of divisions (around 20-30), which is followed by cell death. Nearly, 100 yeast genes have been identified as involved in ageing and whose deletion enhances RLS (Kaeberlein et al. 2005; Longo et al. 2012). One of the best understood replicative ageing pathways involves the gene SIR 2 encoding a member of the sirtuin family of NAD ${ }^{+}$-dependent deacetylases. Overexpression of SIR 2 was shown to extend yeast RLS (Kaeberlein et al. 1999) among other mechanisms, by suppressing homologous recombination of rDNA that leads to the formation of extrachromosomal rDNA circles (ERCs) (Fig. 9.1). These ERCs were thought to limit the mother's cells RLS due to their self-replicating capacity and asymmetrically segregation to the mother cells (Sinclair and Guarente 1997). Recently, this idea is being challenged by the suggestion that rDNA instability, rather than ERCs, is the primary cause of mother cells' senescence and death (Lindstrom et al. 2011). Importantly, mutations that suppress the rDNA instability such as deletion of $F O B 1$, an rDNA replication fork block protein (Kaeberlein et al. 1999), are able to overcome the decreased RLS of sir2 $\triangle$ mother cells (Longo et al. 2012). Several groups have later demonstrated that overexpression of Sir2 homologs, Sir2.1 in Caenorhabditis elegans and dSir2 in Drosophila melanogaster, extends life span (Rogina and Helfand 2004; Tissenbaum and Guarente 2001). Therefore, the first highly conserved determinant of ageing, Sir2, was discovered in yeast.

The role of Sir2 in the regulation of the RLS is not restricted to the formation of ERCs and rDNA instability. Deletion of SIR 2 results in a defect in the asymmetric retention of damage, particularly of oxidatively damaged cytoplasmic proteins in the mother cell leading to a shorter RLS of daughter cells (Aguilaniu et al. 2003). This defective asymmetric retention of damage promoted by deletion of SIR 2 can be overcome by the overexpression of HSP104 (Erjavec et al. 2007) (Fig. 9.1).

Sir2 has also been shown to be fundamental to the regulation of the epigenetic modifications to histones. The best example is the increase in the H4K16 acetylation concurrently with a decline in Sir2 levels during ageing (Dang et al. 2009). Furthermore, Sir2 can also control RLS in an ERC-independent manner, by controlling cytoskeleton function and polarity (Liu et al. 2010). Importantly, overexpression of SIR2 orthologs in worms and flies promotes longevity and activation of the mammalian Sir2-ortholog, SIRT1, can enhance health span in mice (reviewed in Finkel et al. (2009)). Although these results have been questioned by many, there appears to be a general consensus that SIRT1 interacts with important ageing-related pathways in mammals.

Mitochondrial function also plays a critical role in RLS determination, as mitochondrial oxidative phosphorylation (OXPHOS) deteriorates and mitochondrial ROS 


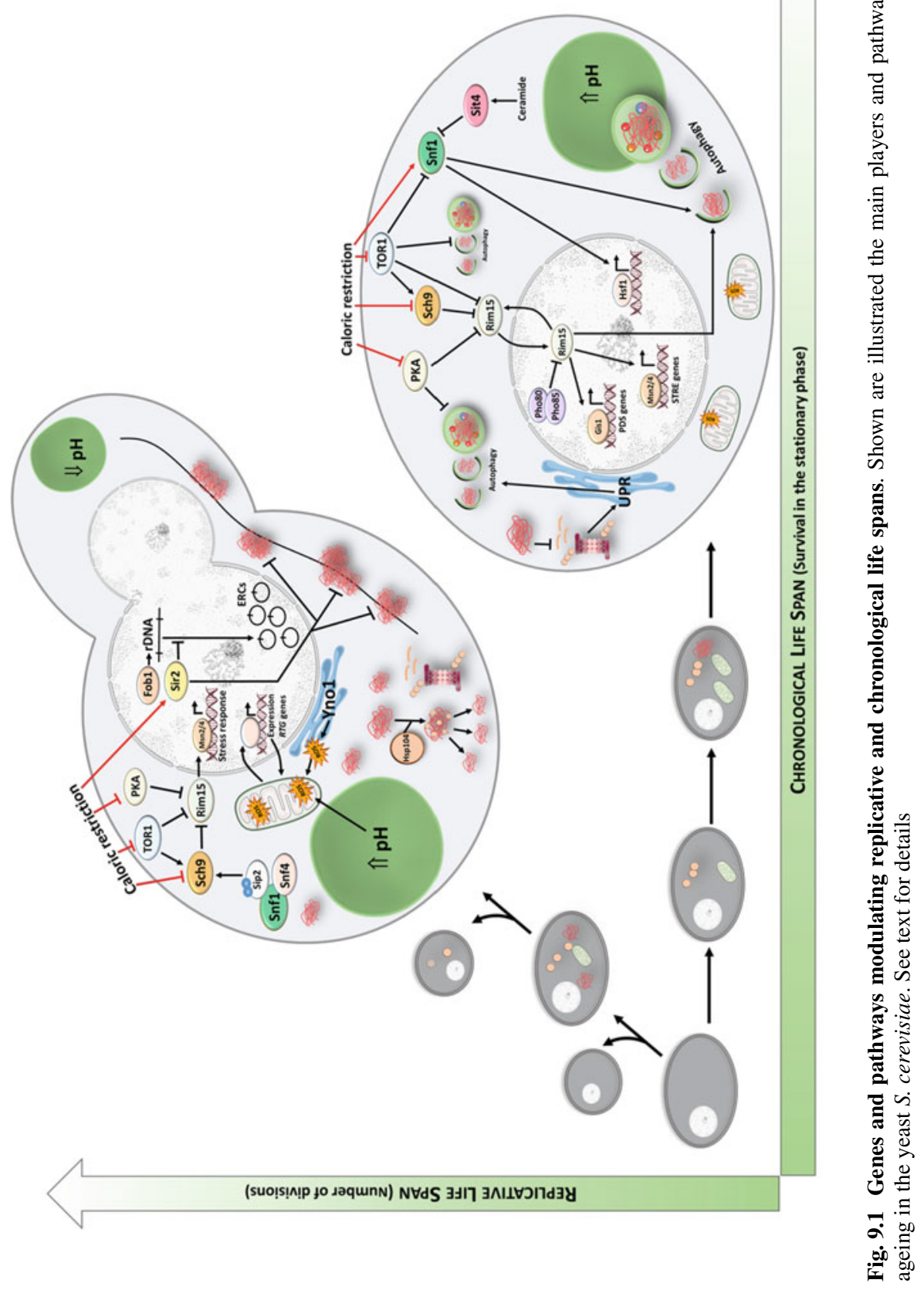


generation increases with age. Jazwinski and colleagues have demonstrated that induction of the retrograde response pathway, which transmits signals of mitochondrial stress to the nucleus, extends RLS in certain genetic backgrounds (Kirchman et al. 1999). Therefore, RLS is extended by enhancement of mitochondrial biogenesis, correct mitochondrial segregation and inheritance, prevention of mitochondrial proteotoxic stress, and maintenance of proper nuclear-mitochondrial communication through activation of mitochondrial retrograde signalling pathways (Fig. 9.1; reviewed in Ruetenik and Barrientos (2015)). Recently, it was shown that the shortening of RLS due to mitochondrial dysfunction was not related to the accumulation of ERCs, but to increased ROS generation of the ER-localized NADPH oxidase Yno1 (Yi et al. 2018). Furthermore, mitochondrial function is also impaired due to the decline of vacuolar acidity that occurs with age. The age-dependent increase of $\mathrm{pH}$ promotes the storage of amino acids in the mitochondria that consequently affects membrane potential and mitochondrial function (Fig. 9.1) (Hughes and Gottschling 2012).

Recently, studies indicate that as cells age, damaged protein aggregates and oxidatively damaged organelles, as mitochondria, are predominantly sequestered in mother cells contributing as ageing factors (Aguilaniu et al. 2003; Denoth Lippuner et al. 2014; Erjavec and Nystrom 2007; Lam et al. 2011; Liu et al. 2010; McFaline-Figueroa et al. 2011). In contrast, disruption of endoplasmic reticulum diffusion barriers can result in the segregation of misfolded protein aggregates into daughter cells (HiguchiSanabria et al. 2014), see for review Smith and Schneider (2018).

The abovementioned detrimental age-dependent changes can be mitigated by anti-ageing intervention such as caloric restriction (CR). Mounting evidences demonstrated that CR extends life span and health span in several model organisms (Fontana et al. 2010). In yeast, CR is achieved by reducing glucose concentration from 2 to $0.5 \%$ or below (Lin et al. 2000). The SIR 2 gene and functional NAD ${ }^{+}$salvage genes were reported to be required for CR-mediated RLS extension (Anderson et al. 2002, 2003; Lin et al. 2000). Although it is still debated the role of Sir2 on the CR-mediated RLS extension, it has been accurately established that CR can also extend life span via Sir2-independent mechanisms and that Sir2 and CR work in parallel pathways (Kaeberlein et al. 2004). Additional discussions of the role of Sir2 and the other yeast sirtuins in RLS extension promoted by CR can be found in recent reviews of this topic Kaeberlein (2010), Kaeberlein and Powers (2007). Curiously, enhanced proteasome activity is able to increase RLS by a mechanism that is genetically distinct from both CR and Sir2 (Kruegel et al. 2011).

Accumulated evidence indicates that CR-mediated RLS extension is largely dependent on reduced Ras-PKA and TOR/Sch9 pathways signalling that play a concerted role in regulating growth, metabolism and stress resistance in response to nutrient availability (Kaeberlein et al. 2005). Importantly, Sch9 activity can be regulated independently of CR to influence RLS through acetylation of Sip2, a component of the yeast AMP-activated protein kinase complex, Snf1 (Lu et al. 2011). These nutrient and energy sensing pathways play a similar role in modulating yeast CLS, as well as longevity in worms, flies and mice, providing strong evidence for their conserved effects on ageing throughout eukaryotes (Fontana et al. 2010). 
CLS defines the survival of stationary-phase cells after depletion of nutrients, and glucose is commonly the first limiting nutrient. Importantly, two main cell populations could be defined when glucose is exhausted at the diauxic shift. One of these populations corresponds to quiescent (Q) cells that are in G0, a non-proliferative state. Q cell population is mainly composed of unbudded daughter cells (Aragon et al. 2008; Li et al. 2013; Miles et al. 2013; Werner-Washburne et al. 2012) that are highly resistant to stress and present rigid cell walls and high accumulation of glycogen and trehalose and low accumulation of reactive oxygen species (ROS). These cells are able to re-enter mitosis when nutrients become available (Aragon et al. 2008; Leonov et al. 2017; Miles et al. 2013). Another cell population is composed of nonquiescent (NQ) cells, most or all of which are first-generation and higher generation mother cells (Aragon et al. 2008; Li et al. 2013; Miles et al. 2013; Werner-Washburne et al. 2012). These NQ cells can be metabolically active, with or without clonogenic capacity, or may exhibit hallmarks of apoptosis or necrosis (Aragon et al. 2008; Li et al. 2013; Miles et al. 2013; Werner-Washburne et al. 2012).

CLS is controlled by a complex signalling network including TORC1 (target of rapamycin complex 1), a highly conserved serine/threonine protein kinase complex that is the major regulator of the signalling network controlling cell growth; PKA (protein kinase A), a major regulator of metabolism, proliferation and stress resistance; and the protein kinase Sch9, a serine/threonine protein kinase that plays a central role in nutrient-mediated signalling (Smets et al. 2010). These three kinase complexes are the so-called ménage-à-trois that integrates inputs from several nutrientsensing systems to regulate metabolism, intracellular trafficking, proteome integrity, autophagy, stress resistance, cell size, progression, growth and sporulation (reviewed in Deprez et al. (2018)). In addition to these regulators, other energy and sensing pathways play important roles in regulating longevity. This is the case for Snf1 (sucrose non-fermenting, protein 1), a member of the conserved AMP-activated protein kinase (AMPK) family that is a major sensor of cellular energy levels, and the Pho85 (phosphate metabolism, protein 85), which together with Pho80 forms a kinase complex with a major role on the cellular response to changes in extracellular and/or intracellular phosphate levels.

These nutrient-sensing pathways may overlap and create redundancy in the modulation of many downstream effector proteins including (among others) Rim15, a serine/threonine protein kinase that is essential for cell cycle arrest at G1 and entry of cells into quiescence. Rim15 is regulated by the TORC1, Sch9, PKA and Pho85 (reviewed in Leonov et al. (2017, Sampaio-Marques et al. (2014a), Smets et al. (2010)). Yak1 is another serine/threonine protein kinase under the control of PKA, and it is required for cell cycle arrest at G1 (reviewed in Leonov et al. (2017), Sampaio-Marques et al. (2014a), Smets et al. (2010)). TORC1 and PKA also control Mck1, a dual-specificity serine/threonine and tyrosine protein kinase. Msn2/4 and Gis 1 are also downstream effectors of these two kinases. These transcription factors are controlled by TORC1 and PKA, as well as by Snf1-they activate the expression of genes involved in stress response and diauxic transition (reviewed in Leonov et al. (2017), Smets et al. (2010)). Another essential transcription factor is Hsfl, which is controlled by Snf1 and is involved in the expression of many genes, particularly those 
related to control of proteostasis and energy generation. Snf1 and TORC1 regulate the transcriptional factor Gln3, which regulates genes involved in the metabolism of nitrogen, and eIF $2 \alpha$, a factor involved in the initiation of protein synthesis. It is also important to highlight the regulation of the Atg1-Atg13 complex, controlled by TORC1, PKA, Snf1 and Pho85, which initiates autophagy by enabling phagophore assembly site (PAS) formation (reviewed in Leonov et al. (2017)). Globally, these downstream effectors enhance several protective systems including glycogen and, glycerol and antioxidant enzymes and mechanisms related to the maintenance of proteostasis, such as HSPs and autophagy (Sampaio-Marques et al. 2014b).

Despite the different mechanisms of life span extension promoted by the inactivation of these signalling pathways, autophagy seems to be a common denominator. Reduced TOR signalling induces autophagy (Noda and Ohsumi 1998), while deletion of $S C H 9$, a TOR effector that can function independently, has a minor impact on autophagy. Nevertheless, the role of Sch9 on vacuole acidification has to be considered, as deletion of SCH9 could extend both RLS and CLS by contributing to the maintenance of vacuole acidification in aged cells (reviewed in Ruckenstuhl et al. (2014), Tyler and Johnson (2018a)). These nutrient-sensing pathways together with PKA are crucial for the regulation of $\mathrm{pH}$ homeostasis through their influence on the proton pumping activity of the V-ATPase, and possibly also on Pma1, from the plasma membrane ATPase (Deprez et al. 2018). $\mathrm{pH}$ homeostasis is a crucial regulator of autophagy, as the final step of autophagy is linked to vacuolar membrane integrity and acidification of the vacuolar lumen (reviewed in Deprez et al. (2018)). Besides PKA's role in $\mathrm{pH}$ homeostasis, inactivation of PKA also induces autophagy associated with RLS and CLS extension, albeit less efficiently than autophagy induction promoted by TOR inactivation (Budovskaya et al. 2004) or by deletion of both $\mathrm{SCH} 9$ and PKA (Yorimitsu et al. 2007). In contrast, the positive regulator of autophagy, Snf1, promotes a reduction of CLS when deleted (Wang et al. 2001).

Regarding Sir2, its role on CLS is far more complex. Depending on the strain background and growth media, deletion of SIR2 either has no effect or induces a moderate increase of CLS (reviewed in Wierman and Smith (2014)). Consequently, Sir2 has been mainly assigned a pro-ageing role in CLS (reviewed in SampaioMarques et al. (2014a)). Although Sir2 might antagonize CLS extension promoted by CR (Fabrizio et al. 2005), it was also shown that CR extends CLS independently of the sirtuins including Sir2 (Smith et al. 2007). We have shown that autophagy maintenance at homeostatic levels promoted by CR or TOR1 deletion is achieved by decreasing Sir2 levels and activity (Guedes et al. 2017). Although SIR2 deletion does not have a major effect on CLS, it does compromise the extension of CLS observed in $\mathrm{SCH} 9$ deleted cells and in cells treated with the life span-promoting agent resveratrol (Fabrizio et al. 2005; Howitz et al. 2003). Furthermore, Sir2 plays an important role in autophagy regulation during CLS in certain scenarios. We have previously shown that Sir2, similar to mammalian SIRT1, activates autophagy and mitophagy through the transcriptional regulation of ATG8 and ATG32 under proteotoxic conditions (Sampaio-Marques et al. 2012). Therefore, it is tempting to speculate that Sir2 supports life span extension of $\mathrm{SCH} 9$ deleted cells by maintaining autophagy. 
Besides the activation of a general stress response, the pro-longevity effects linked to reduced activity of nutrient-sensing pathways appears to be also associated with ROS signalling and increased mitochondria function. Our studies have shown that abrogation of catalase activity or of nutrient-sensing pathways by CR extends CLS by producing hydrogen peroxide, which leads to the activation of superoxide dismutases that inhibit the accumulation of superoxide anions (Mesquita et al. 2010). These findings established a role for hormesis effects of hydrogen peroxide in promoting longevity. Later, it was reported that during exponential-growing phase, TOR 1 or SCH9 deleted cells generate mitochondrial ROS, as an adaptive hormetic signal, which results in the reduction of ROS levels at stationary phase and extension of CLS (Pan et al. 2011). A few years ago, the beneficial effects of hormetic mitochondrial ROS on longevity signal that extends yeast CLS were shown to involve epigenetic alterations and the DNA damage responses (DDR) kinases, Tel1 and Rad53 (Schroeder et al. 2013). This hormetic pathway is independent and distinct from the nuclear DDR and involves histone modifications (Schroeder et al. 2013).

The existence of a nuclear pathway that senses mitochondrial ROS generation/accumulation points to the crucial role of mitochondrial function on ageing. In fact, the longevity-promoting effects of the global activation of general stress response by decreasing the nutrient-sensing pathways' activity appear to be associated with an increase in mitochondria function. In line with this, the lack of mitochondrial respiration severely impacts the longevity of stationary-phase cells (reviewed in Sampaio-Marques and Ludovico (2018)). In contrast, long-lived cells deleted on TORI (Bonawitz et al. 2007; Ocampo et al. 2012; Pan et al. 2011) or SCH9 (Lavoie and Whiteway 2008) presented an increased respiratory capacity. The promotion of longevity encompasses the activation of the Msn2/4 and Gis1 stress response (Fig. 9.1) (Ewald et al. 2016) and the Rph1-dependent epigenetic silencing by triggering a non-canonical activation of the DDR pathways (Schroeder et al. 2013).

More recently, novel ageing determinants were identified, as proteins involved in chromatin remodelling (Swr1, Arp6 and Swc3), Arv1, a lipid homeostasis factor that modulates autophagy, Tep1, the homologue of the human tumour suppressor PTEN, and proteins associated with phosphatidylinositol phosphate metabolism (Garay et al. 2014). A connection between sphingolipids and cell signalling through TOR, Sch9 and the ceramide-activated protein phosphatase Sit4 was recently shown to impact on mitochondria function, autophagy and CLS (Vilaca et al. 2018) (Fig. 9.1). Details on the link between sphingolipids signalling and CLS can be found in (Oliveira et al. 2017).

Numerous studies point to distinct determinants of yeast RLS and CLS. For example, deletion of SIR2 or RAS2 has dissimilar effects on RLS and CLS (reviewed in Smith and Schneider (2018)). In contrast, both RLS and CLS are extended in response to $\mathrm{CR}$ and other interventions that abrogate nutrient-sensing pathways. Nevertheless, it remains unclear whether similar downstream molecular events are common to both yeast ageing paradigms (Sampaio-Marques et al. 2014a). Importantly, both yeast ageing paradigms are connected. As briefly mentioned above, chronologically aged yeast cells show a proportional reduction in RLS (Ashrafi et al. 1999; Murakami et al. 2012; Piper et al. 2006) that is prevented by CR, suggesting that the metabolic 
state and mitochondrial function of stationary-phase cells determine their replicative potential upon transfer to growth conditions (Delaney et al. 2013).

\subsection{Proteostasis and Yeast Ageing}

Ageing is driven by accumulation of damage in highly conserved cell-intrinsic processes such as chromosome structure/organization, transcriptional regulation, nuclear export/import, protein translation and quality control, recycling of damage/unnecessary organelles, maintenance of cytoskeletal structure and extracellular signalling (DiLoreto and Murphy 2015). These processes have the ability to communicate with each other resulting in an intricate interplay that governs cells' ageing. Therefore, the knowledge of the specific cellular and molecular mechanisms underlying ageing represents one of the most complex issues that have yet to overcome.

Several studies focused on molecular alterations occurring during yeast ageing revealed a series of progressive events that collectively contribute to ageing phenotypes. These events integrate damage and dysfunction with stress pathways, including oxidative stress associated with mitochondrial dysfunction and accumulation of ROS, genomic instability associated with nuclear DNA damage, mutagenesis and replication stress, metabolic alterations and loss of proteostasis. Importantly, DNA damage and error-prone DNA repair systems have been assumed as key for the mechanisms behind age-dependent genomic instability observed during ageing. The results of several studies consistently point to a role for oxidative damage that induces senescence and cell death as an important determinant of life span (reviewed in Weinberger et al. (2013)). However, the relationships between ROS, ageing and age-related diseases suggest increased complexity in this scenario (Ludovico and Burhans 2014; Weinberger et al. 2013).

Protein quality control systems as autophagy play a key role in the DDR by controlling the levels of proteins involved in cell cycle checkpoints and DNA synthesis/repair mechanisms. For example, in S. cerevisiae, DNA damage induces the autophagic degradation of ribonucleotide reductase 1 (Rnr1) (Dyavaiah et al. 2011), the large subunit of ribonucleotide reductase (RNR), which is a highly conserved enzymatic complex catalysing the formation of deoxyribonucleotides required for both DNA replication and repair. This DDR-dependent autophagic pathway in yeast was called genotoxin-induced targeted autophagy (GTA) and requires the involvement of the DDR kinases, Mec1 and Rad53, as well as a central component of the selective autophagy machinery, Atg11 (Eapen et al. 2017). Recently, it was reported that the kinase Mec1 plays a fundamental role in protein homeostasis (CorcolesSaez et al. 2018). In agreement, it is becoming well recognized that one of the major determinants of ageing is proteostasis and that the other ageing hallmarks are intimately related to it. It is the example of the nutrient-sensing pathways that when inactivated mainly contribute to the maintenance of the proteome during ageing (Sampaio-Marques et al. 2014a). In this sense, it is proposed that early changes on protein homeostasis network that result in the cellular loss of proteostasis could 
be one of the earliest events dictating ageing progression, affecting a multitude of downstream processes (Labbadia and Morimoto 2014). In fact, cells have multiple stress-responsive mechanisms to combat loss of proteostasis associated with cellular ageing as described below.

The ability of cells to maintain protein homeostasis, or proteostasis, in response to intrinsic cellular and environmental insults, which accumulate over time, is one of the main determinants of life span (Morimoto and Cuervo 2014). Proteostasis, referred as the healthy maintenance of the cellular proteome, comprises highly complex and interconnected pathways that govern the fate of proteins. Proteostasis is controlled by a multi-compartmental system that has the ability to coordinate protein synthesis, processing, trafficking, folding, localization, assembly/disassembly and degradation (Sampaio-Marques and Ludovico 2018). A major determinant of loss of proteostasis and protein aggregation is the overproduction and accumulation of unstable proteins (Lopez-Otin et al. 2013). For example, it was demonstrated that inhibition of protein translational machinery with cycloheximide blocks the formation of protein aggregates indicating that active protein translation is required for stress-induced protein aggregation in yeast (Zhou et al. 2014). Although the mechanisms underlying this observation remain unclear, a reduction in handling the burden of newly translated unfolded proteins and an increase in free molecular chaperones as well as in the activity of the degradation pathways could be simple explanations (Medicherla and Goldberg 2008).

Molecular chaperones assist in the folding/refolding of proteins (Fig. 9.2). Chaperones can be found in the cytoplasm but also in the ER and mitochondria. A recent study revealed that decline in chaperone activity in each cellular compartment triggers a response in other compartments that result in loss of respiration capacity, demonstrating the dependence of mitochondrial activity on cell-wide proteostasis. This phenomenon has been called cross-organelle stress response (CORE) and has a protective role by extending both CLS and RLS (Peric et al. 2016). Although several physical organelle contact sites exist in yeast, their involvement in CORE and the cell-wide proteostasis system is yet to be elucidated. Importantly, it was demonstrated that ER-formed protein aggregates are frequently associated with or are later captured by mitochondria (Zhou et al. 2014). In line with this concept, aged replicative cells exhibit a gradual decline of aggregate-mitochondria association decreasing mobility and leakage of aggregates from mother into the bud contributing to the decreased life span of daughter cells (Zhou et al. 2014).

The activity of ATP-dependent chaperones is greatly affected by the agedependent reduction of cellular energy, due to reduced mitochondrial functionality and deregulation of lipid and glucose metabolism (Ma and Li 2015; Ritz and Berrut 2005). The activity of molecular chaperones could also be affected by their availability, which does not meet the needs of aged cells. These phenomena might be aggravated by protein modifications that are enhanced during ageing, such as accumulation of advanced glycation end products through non-enzymatic modifications that interfere with the chaperone's ability to recognize the target (Vanhooren et al. 2015), resulting in the accumulation and aggregation of the defective proteins (Kumar et al. 2007). Consistent with this possibility, it was shown that downregulation of 


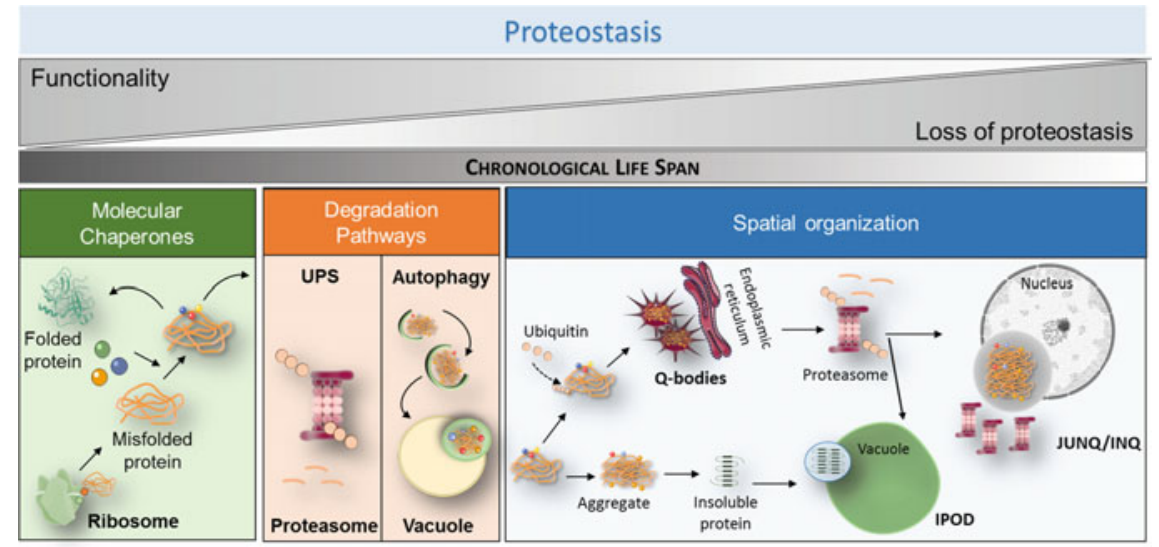

Fig. 9.2 Proteostasis and yeast ageing. Proteostasis is mainly maintained by the action of molecular chaperones and the two degradation pathways, the ubiquitin-proteasome system (UPS) and autophagy. In addition, yeast cells have developed a spatial quality control system where misfolded and aggregated proteins are sequestered into larger protective inclusions: the juxtanuclear quality control site (JUNQ)/the intranuclear quality control compartment (INQ) and the peripheral vacuole-associated, insoluble protein deposit (IPOD). See text for more details

yeast Hsp90 activity results in an increase in heat shock protein synthesis due to the inability to efficiently repress Hsf1 (Duina et al. 1998; Harris et al. 2001), which is correlated with increased viability over time. Thus, an enhancement of chaperone activity is associated with increased longevity. However, age-mediated alterations in proteostasis are due not only to decreased chaperones activity, but also to alterations in degradation pathways, the ubiquitin-proteasome system (UPS) and autophagy (Fig. 9.2). In yeast chronological ageing, proteasome dysfunction occurring over time induces the accumulation of protein aggregates and formation of inclusions that further obstruct proteasome function in a vicious cycle (Andersson et al. 2013). Our experimental results showed an accumulation of ubiquitinated proteins associated with an increase in levels of RPN4 along chronological ageing, reflecting an impairment of proteasome activity during yeast ageing (Sampaio-Marques and Ludovico 2018).

Proteasome dysfunction might also be potentiated by the sequestration in protein aggregates of factors required for proteasome activity, such as ubiquitin ligases/proteases or proteasome activators (Andersson et al. 2013). For example, the production and accumulation of ROS lead to proteasome activity reduction and subsequent accumulation of carbonylated proteins and specific modifications in certain proteins, such as E1 and/or E2 enzymes, which results in the impairment of the ubiquitin-binding (da Cunha et al. 2011). In contrast, overexpression of key molecules, such as the proteasome chaperone Ump1, results in the proteasomemediated protein degradation enhancement with the consequent longevity extension (Chen et al. 2006). 
Accumulating evidence also suggests that damaged proteins are not randomly distributed in the cell during ageing. Yeast cells have developed a spatial quality control system where misfolded and aggregated proteins are sequestered into larger protective inclusions (Alvers et al. 2009a) (Fig. 9.2). This spatial compartmentalization of protein aggregates is a complementary protein quality control strategy that acts in parallel with temporal quality control. The presence of these inclusions is not essential for their degradation, but it may facilitate refolding/degradation by increasing the proximity of chaperones and their substrates limiting the toxic interactions of misfolded proteins. Several studies suggest that immediately upon misfolding, an active chaperone-dependent transport of damaged proteins to dynamic compartments called Q-bodies, which are attached to the ER, takes place (Escusa-Toret et al. 2013). The damaged proteins inside of these Q-bodies are rapidly cleared through the UPS-however, if clearance is impaired, these misfolded proteins concentrate in one of the two major protein quality control compartments, the juxta nuclear quality control site (JUNQ) and the peripheral vacuole-associated, insoluble protein deposit (IPOD) (Kaganovich et al. 2008) (Fig. 9.2). More recently, JUNQ was found inside the nucleus, where it serves as a new intranuclear quality control compartment (INQ) for the deposition of both nuclear and cytosolic misfolded proteins, irrespective of ubiquitination (Miller et al. 2015). Proteins are targeted to the JUNQ by a ubiquitin-based sorting mechanism, while they are sent non-ubiquitinated to IPOD (Kaganovich et al. 2008) (Fig. 9.2). Recently, it was found that the small Hsp42 can assemble into versatile dynamic oligomers - the Hsp42-containing stationaryphase granules (Hsp42-SPGs), which contain protein components including molecular chaperones, metabolic enzymes and regulatory proteins (Lee et al. 2018). These Hsp42-SPGs are enriched in long-lived quiescent cell populations, suggesting that these granules may help quiescent cells to combat various stresses during stationary phase, by mechanisms that remain unclear (Lee et al. 2016). Furthermore, Hsp42SPGs may work as centres that control both protein quality and quantity in stationaryphase cells (Lee et al. 2018).

Like the other proteostasis pathways, spatial quality control also declines with age, and cells that lack this ability show accelerated ageing (Escusa-Toret et al. 2013). During ageing, the increase in the load of aggregated proteins and the inactivation of cellular chaperones could provide one explanation for the age-associated loss of spatial protein quality control (Hill et al. 2017). Furthermore, the reduction on efficiency of this spatial protein quality control system might be also related with the failure of organelles function. For example, vacuolar $\mathrm{pH}$ alterations could cause a breakdown in vesicle trafficking and fusion to the vacuole, an important process for spatial sequestration of aggregated proteins. Furthermore, disruption of a functional actin cytoskeleton might also affect vesicle trafficking and fusion, mitochondrial inheritance, and increase ROS accumulation - this could explain the inefficient inclusion formation observed in ageing cells (reviewed in Hill et al. (2017)).

Surveillance of proteostasis is mainly played by the chaperones and the main protein degradation pathways, the UPS and the autophagy. Although UPS is the primary cellular route for protein degradation, it does not allow for the degradation of unfolded or large protein complexes. Therefore, larger substrates, such as 
large protein inclusions, can be directly degraded by autophagy. In the budding yeast $S$. cerevisiae, macro(autophagy) occurs through the formation of a doublemembrane vesicle-autophagosome-that sequesters cytosol and organelles and fuses with the vacuole releasing the content to be degraded and recycled. Besides bulk unspecific degradation, autophagy can occur by selective mechanisms encompassing the degradation of specific cargos such as organelles (reviewed in Galluzzi et al. (2017)). Selective autophagy requires functional actin cytoskeleton for specific degradation of mitochondria, peroxisomes, mature ribosomes, and cytosolic proteins such as acetaldehyde dehydrogenase Ald6 (reviewed in Smith and Schneider (2018)). The functioning of autophagy is supported by two main groups of genes, autophagy-related genes (ATG) and vacuolar protein sorting (VPS) genes (Reggiori and Klionsky 2013; Tyler and Johnson 2018b). Collectively, the processes underlying autophagy are highly complex and beyond the scope of this chapter and have been extensively reviewed elsewhere Yin et al. (2016).

Similar to UPS, autophagy activity decreases during ageing, as reported in different model systems (reviewed in Rubinsztein et al. (2011)). However, due to the complexity of the autophagy process and the stochastic nature of ageing, the mechanisms underlying decreased autophagy remain largely unclear. The decline of autophagy activity promoted by ageing enhances the accumulation of aberrant proteins/aggregates, causing additional molecular and cellular damage, as a vicious cycle. Furthermore, an age-associated increase of vacuolar $\mathrm{pH}$ (Fig. 9.1), which limits the activities of vacuolar proteases and results in the loss of vacuolar homeostasis, may contribute to autophagy impairment during ageing (Nakamura et al. 1997). Autophagy deregulation in aged cells can also be a consequence of persistent activity stimulation. Although increased autophagy might initially have a favourable outcome, if maintained at a high rate, it can promote the depletion of functional organelles/proteins and essential autophagic molecules, contributing to cell death and thus shortening of life span (Meijer and Codogno 2007; SampaioMarques et al. 2012). In agreement, we showed that heterologous expression of human alpha-synuclein (aSyn) in yeast cells results in aberrantly high activation of autophagy associated with shortening of CLS (Sampaio-Marques et al. 2012). Furthermore, all the genetic and environmental manipulations reducing aSyn toxicity resulted in decreased autophagy activity (Guedes et al. 2017; Sampaio-Marques et al. 2012) indicating that autophagy should be maintained under homeostatic levels. It is, nonetheless, well recognized that autophagy is required for maximal CLS and has been implicated in almost all the CLS promoting interventions, as discussed herein and reviewed in Sampaio-Marques et al. (2014a), Tyler and Johnson (2018a). Different genetic studies have demonstrated that genes encoding proteins involved in autophagy machinery as ATG1, ATG2, ATG7, ATG8, ATG16 or VPS21 are required for life span extension (Alvers et al. 2009a; Aris et al. 2013; Fabrizio et al. 2010; Matecic et al. 2010). Dietary interventions such as caloric or methionine restriction were also shown to be dependent on autophagy (Aris et al. 2013; Ruckenstuhl et al. 2014). As we have discussed in the previous section, nutrient-sensing pathways also have profound effects on autophagy and longevity, as well as other relevant players linking metabolism, autophagy and longevity. Notably, accumulation of acetyl- 
CoA, a critically important molecule in metabolism, has been shown to result in the hyperacetylation of histones that transcriptionally repress autophagy genes and negatively impact ageing (Eisenberg et al. 2014). The Esa1 and Rpd3 antagonistic acetyltransferase-deacetylase pair has also been shown to transcriptionally regulate autophagy_implicating epigenetic regulation of autophagy also in RLS (Yi et al. 2012). Together, these studies clearly demonstrate a bona fide role for epigenetics in the regulation of autophagy and yeast life span.

Lipid metabolism can also engage the autophagy machinery to positively regulate longevity, as referred above. The Arv1, a protein involved in sterol and sphingolipids metabolism, was identified as involved in the regulation of CLS through autophagy (Garay et al. 2014). An additional study demonstrated that supplementation of nutrient medium with phosphatidylethanolamine (PE) or genetic interventions that result in increased PE levels activate autophagy and extend CLS (Rockenfeller et al. 2015).

Interestingly, due to the large number of conserved ageing-related genes and ageing mechanisms in yeast and humans, testing candidate anti-ageing molecules in yeast has proven highly successful in the search for potential anti-ageing therapies. Yeast-based studies have helped to understand the mode of action of anti-ageing molecules such as rapamycin, spermidine or resveratrol. Rapamycin is a macrolide antibiotic with antifungal and immunosuppressive properties, which inhibits the TOR signalling pathway. Autophagy activation was shown to be essential for rapamycinmediated life span extension in yeast (Alvers et al. 2009b). The polyphenol resveratrol and the natural polyamine spermidine are currently the most promising potential anti-ageing agents that were discovered in yeast and shown to activate autophagy and extend both RLS and CLS (Eisenberg et al. 2009; Morselli et al. 2011). Collectively, these observations are consistent with results from the large number of studies that point to general anti-ageing properties of autophagy. In spite of the role of autophagy in CLS, autophagy does not appear to contribute to RLS under normal growth conditions. Indeed, deletion of most of the $A T G$ genes has negligible effects on RLS, and in some cases even results in extension of RLS (Ghavidel et al. 2015; McCormick et al. 2015).

Altogether, these studies demonstrate that yeast is an invaluable tool for the identification and characterization of conserved mechanisms that promote cellular longevity, and that autophagy plays an important role in nearly all known longevitypromoting interventions, as reviewed elsewhere Tyler and Johnson (2018a).

\subsection{Modelling Age-Related Diseases in Yeast}

Deregulation of protein network functionality is correlated with ageing and is a major risk factor for the development of a wide spectrum of age-related protein diseases (Morimoto and Cuervo 2014). The budding yeast $S$. cerevisiae is a simple unicellular eukaryotic organism that shares well-conserved molecular and cellular mechanisms with higher eukaryotes and has been particularly useful as a biological model for ageing and age-related diseases (Tenreiro et al. 2013). Thus, S. cerevisiae 
has played an extremely important role in the discovery of key molecular events associated with neurodegenerative diseases, including Parkinson's (PD), Alzheimer's (AD) and Huntington's (HD) diseases. These protein misfolding disorders are agerelated degenerative diseases in which misfolded proteins are prone to form intra- or extracellular aggregates with specific composition and localization for each disease. While intracellular aSyn and huntingtin $(\mathrm{Htt})$ aggregates are hallmarks of PD and HD, respectively, extracellular aggregates of tau protein and amyloid- $\beta(\mathrm{A} \beta)$ peptide are characteristic of AD. Depending on the disease, the resulting aggregates might result in the loss of protein function and/or in the gain of a cytotoxic function. Mitochondrial dysfunction, transcriptional dysregulation, trafficking defects and loss of proteostasis are some of the molecular and cellular mechanisms conserved from yeast to human that underlie the pathogenesis of these diseases (reviewed in Tenreiro and Outeiro (2010)).

It is estimated that around $25-30 \%$ of the genes linked to human diseases have yeast orthologues (Bassett et al. 1996). Thus, if a gene related to a human disease has a yeast homologue, its role can be investigated by simply deleting or overexpressing this gene in yeast. For example, yeast-based studies on the SODI and YHF1 genes, homologs of the human genes involved in Friedreich's ataxia and amyotrophic lateral sclerosis, respectively, contributed greatly to our understanding of these disorders. Furthermore, even if a human gene is absent from the yeast genome, its role in disease can be modelled by the heterologous expression of the human gene in yeast cells. Yeast models for PD, HD and AD, which are examples of this strategy, have been extensively exploited and have greatly contributed to the elucidation of the molecular and cellular aspects of these disorders, as detailed below (Miller-Fleming et al. 2008; Sampaio-Marques and Ludovico 2015; Sampaio-Marques and Ludovico 2018).

A yeast HD model can be generated by the heterologous expression of mutant human Htt exon 1 with different polyglutamine (polyQ) expansions (more than 35 glutamine residues) in yeast cells, reproducing many of the cellular and molecular features of HD pathology. For example, expression of mutant fragments of Htt resulted in polyQ length-dependent aggregation and toxicity, endocytosis impairment, transcriptional dysregulation, mitochondrial dysfunction, oxidative stress and apoptosis (reviewed in Tenreiro and Outeiro (2010)). Furthermore, genetic screens using yeast HD models have identified different modulators of mutant Htt aggregation and toxicity. Notably, chaperones members of Hsp40 and Hsp70 families have been identified as potential therapeutic targets (reviewed in Tenreiro and Outeiro (2010)). Yeast HD models have also been useful in screening drugs.

Studies on AD primarily make use of human cell lines and transgenic mouse models. However, yeast AD models are becoming increasingly important to unravel fundamental molecular aspects of AD. Pathological hallmarks of this disease include the presence of extracellular plaques of $A \beta$ and intracellular neurofibrillary tangles of phosphorylated tau protein. Tau and $\mathrm{A} \beta$ have no functional yeast orthologues and thus, different yeast models have assessed the cellular consequences of expressing $A \beta$ peptides or Tau. Different yeast models have been used, and some of them fuse A $\beta 40$ or A $\beta 42$ to C-terminal part of Sup35, a translation termination factor without the prion domain, to create an oligomerization assay to find specific point mutations able to 
inhibit $A \beta$ oligomerization (Bagriantsev and Liebman 2006). These models have been used in high-throughput screens resulting in the identification and validation of two compounds with anti-oligomeric effects (Park et al. 2011). In other yeast AD models, the $A \beta 42$ peptide is directed to the secretory pathway (D'Angelo et al. 2013; Treusch et al. 2011).

Although only a few studies have employed yeast to study the biology of Tau, the data obtained revealed that yeast cells have an enormous potential to disclose key aspects of Tau pathophysiology, since these models recapitulate central features of the AD, including Tau hyperphosphorylation at pathological residues, conformational changes and aggregation (reviewed in Verduyckt et al. (2016)). For example, in yeast, Tau phosphorylation is regulated by the kinases Mds1p and Pho85p, the orthologues of human GSK3b and CDK5, respectively, at the same residues that Tau is hyperphosphorylated in neurons (Vandebroek et al. 2005). Downregulation of Pho85 increases Tau phosphorylation and aggregation, while deletion of MDS1 is associated with reduced Tau phosphorylation (Vandebroek et al. 2005). Furthermore, oxidative stress and dysfunctional mitochondria exacerbate Tau aggregation, although Tau is less phosphorylated under those conditions, suggesting that other mechanisms are involved in Tau aggregation (Vanhelmont et al. 2010) (for review see Seynnaeve et al. (2018)).

Insoluble aggregates of aSyn are found in synucleinopathies including idiopathic and familial forms of PD. Duplication or triplications of SNCA gene, which encodes aSyn, are linked to sporadic PD, while aSyn point mutations (A30P, E46K, H50Q, G51D, A53T and A53E) are associated with familial PD forms, with early onset (reviewed in Sampaio-Marques and Ludovico (2015)). Cell-based models for PD include yeast models, immortalized cell lines, primary neuronal cultures, stem cells and patient-derived cell models. These cellular models have been widely explored to dissect molecular mechanisms behind pathology using unbiased genetic screens, as well as multi-omic approaches to identify relevant genes and proteins. In addition, they can be easily manipulated genetically and pharmacologically at a reduced cost and in the absence of ethical issues. Nevertheless, these cellular models cannot reproduce several features of disease related to multicellularity and require validation in animal models.

The yeast $S$. cerevisiae is one of the best characterized eukaryotic organisms that provides a relevant biological context for the study cellular pathologies associated with PD (reviewed in Tenreiro et al. (2017)). Several molecular aspects of PD have been modelled in yeast, even though yeast lacks orthologs for aSyn. The first PD yeast model was reported in 2003 (Outeiro and Lindquist 2003), and since then a number of different humanized yeast PD models have been developed and employed to investigate PD. Similar to observations in other PD models, aSyn heterologous expression in yeast inhibits cell growth and promotes cell death in a concentration-dependent manner (Outeiro and Lindquist 2003). Independent studies identified mitochondrial dysfunction associated with oxidative stress (Buttner et al. 2008; Sampaio-Marques et al. 2012; Sharma et al. 2006), proteasome impairment (Chen et al. 2005; Sharma et al. 2006), autophagy and mitophagy dysfunction (Petroi et al. 2012; SampaioMarques et al. 2012), vesicular trafficking defects (Outeiro and Lindquist 2003) and 
ER-to-Golgi trafficking impairment (Cooper et al. 2006) as relevant features of PD (Fig. 9.3). Furthermore, several post-translational modifications such as phosphorylation, ubiquitination, sumoylation and acetylation appear to influence aSyn toxicity and inclusion formation (reviewed in Tenreiro et al. (2017)). Our group was the first to develop a yeast model to study aSyn toxicity during ageing. Most yeast PD models are based on the heterologous expression of human aSyn under the control of a strong GAL promoter. Nonetheless, to avoid metabolic manipulations during chronological ageing, the GAL promoter was replaced by the TPII promoter, which results in aSyn expression at lower levels when compared to $G A L$ promoter, but allows for constitutive expression of aSyn during growth and ageing (Fig. 9.3).

Ageing constitutes a major risk factor for neurodegenerative diseases including PD and other synucleinopathies. By exploring the pathobiology of aSyn during yeast ageing, we observed that aSyn-expressing cells display a dramatic increase of autophagy and particularly of mitophagy that is deleterious for cells and shortens life span (Sampaio-Marques et al. 2012). Although increased autophagy can help aSyn clearance in functionally competent cells, it might also affect autophagy efficiency and selectivity in aged cells that have lost proteostasis. Studies in other cellular models have associated aSyn toxicity with aberrantly high activation of autophagy (Choubey et al. 2011; Stefanis et al. 2001; Xilouri et al. 2009). In addition, our experimental results showed that impairment of mitophagy by deletion of the yeast mitophagyspecific genes, ATG11 and ATG32, resulted in CLS extension, further implicating mitophagy in aSyn toxicity. When exploring the pathways underlying autophagy and mitophagy after they have been aberrantly stimulated, we found that deletion of the SIR 2 gene alleviated aSyn toxicity as evidenced by CLS extension, and this phenomenon is linked to a drastic inhibition of autophagy and mitophagy (SampaioMarques et al. 2012) (Fig. 9.3). Notably, Sir2 was determined to be essential for the transcriptional regulation of $A T G 8$ and $A T G 32$ in stationary-phase cells expressing aSyn toxic variants (Sampaio-Marques et al. 2012). Our work emphasizes the fact that increased autophagy/mitophagy activity mediated by Sir2-mediated transcriptional regulation of $A T G$ genes is an important phenomenon linked to aSyn toxicity during ageing.

In support of an association between exacerbated autophagy and aSyn toxicity, we have also shown that interventions that extend longevity and are associated with autophagy regulation, such as CR and inactivation of the TOR signalling pathway, are able to abolish aSyn toxicity and restore normal chronological longevity by maintaining autophagy at homeostatic levels (Guedes et al. 2017). In general, our results strongly suggest that it is important for life span extension to maintain autophagy under homeostatic levels, as has been reported in other biological systems. Furthermore, together with other findings, our data clearly indicate the relevance of proteostasis control in this age-related disease and confirm the utility of yeast as a model system for investigating different aspects of aSyn toxicity.

In conclusion, yeast cell-based models for neurodegenerative diseases provide valuable tools for deciphering the biological mechanisms of pathogenesis of these diseases as well as the discovery of novel therapeutic targets for treating them. Notably, yeast is also a well-recognized cellular ageing model that makes it possible to inves- 

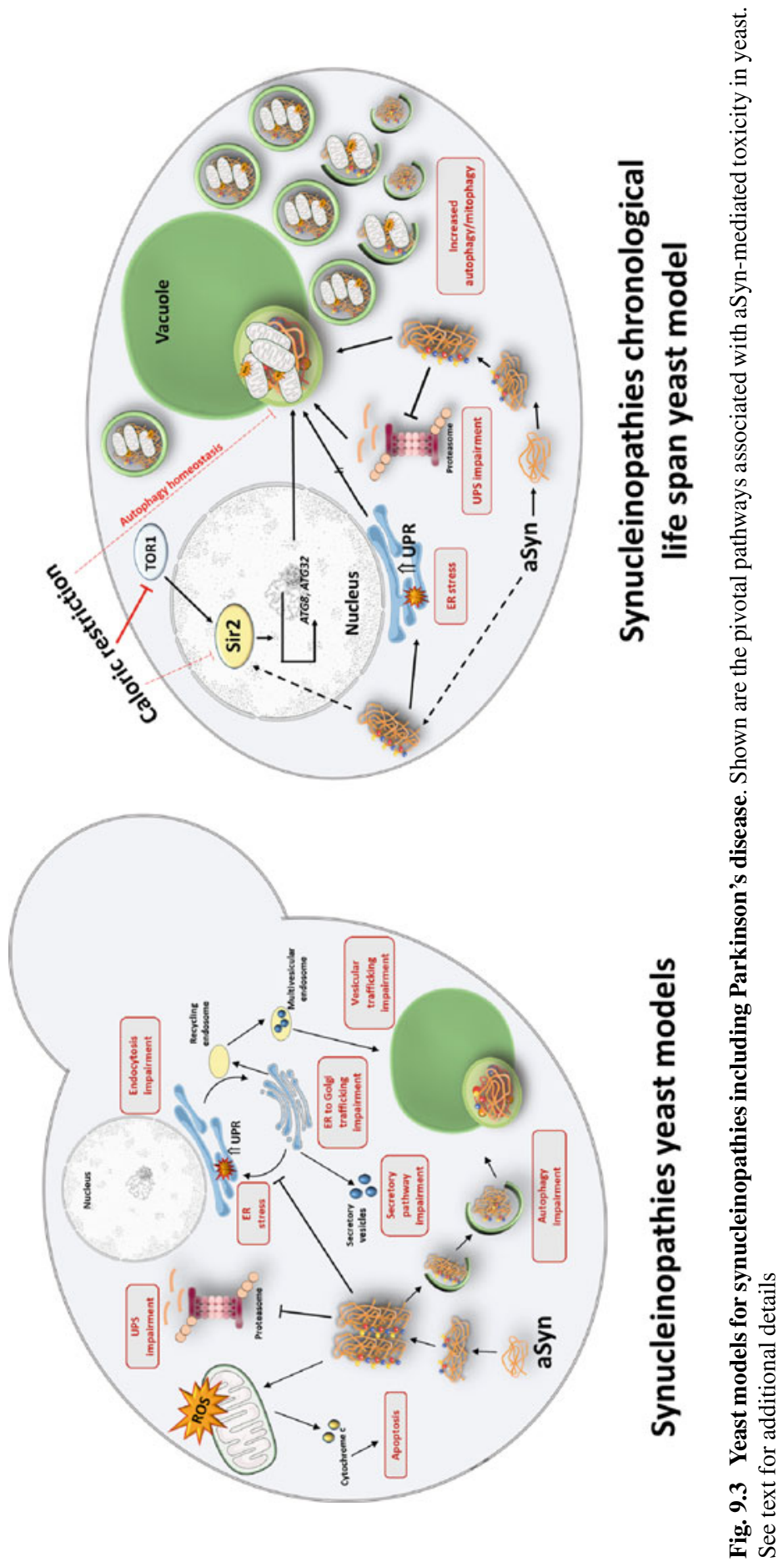
tigate ageing as a component of neurodegenerative and age-related diseases in a manner that may not be possible in other cellular and animal models. To date, yeast ageing models have not been used to assess the toxicity of different factors in either HD or AD. This should provide fruitful avenues of investigation of these diseases in the future, as has been the case for Parkinson's and other synucleinopathies.

\section{References}

Aguilaniu H, Gustafsson L, Rigoulet M, Nystrom T (2003) Asymmetric inheritance of oxidatively damaged proteins during cytokinesis. Science 299:1751-1753

Alvers AL, Fishwick LK, Wood MS, Hu D, Chung HS, Dunn WA Jr, Aris JP (2009a) Autophagy and amino acid homeostasis are required for chronological longevity in Saccharomyces cerevisiae. Aging Cell 8:353-369

Alvers AL, Wood MS, Hu D, Kaywell AC, Dunn WA Jr, Aris JP (2009b) Autophagy is required for extension of yeast chronological life span by rapamycin. Autophagy 5:847-849

Anderson RM et al (2002) Manipulation of a nuclear NAD+ salvage pathway delays aging without altering steady-state NAD+ levels. J Biol Chem 277:18881-18890

Anderson RM, Bitterman KJ, Wood JG, Medvedik O, Sinclair DA (2003) Nicotinamide and PNC1 govern lifespan extension by calorie restriction in Saccharomyces cerevisiae. Nature 423:181-185

Andersson V, Hanzen S, Liu B, Molin M, Nystrom T (2013) Enhancing protein disaggregation restores proteasome activity in aged cells. Aging 5:802-812

Aragon AD et al (2008) Characterization of differentiated quiescent and nonquiescent cells in yeast stationary-phase cultures. Mol Biol Cell 19:1271-1280

Aris JP et al (2013) Autophagy and leucine promote chronological longevity and respiration proficiency during calorie restriction in yeast. Exp Gerontol 48:1107-1119

Ashrafi K, Sinclair D, Gordon JI, Guarente L (1999) Passage through stationary phase advances replicative aging in Saccharomyces cerevisiae. Proc Natl Acad Sci USA 96:9100-9105

Bagriantsev S, Liebman S (2006) Modulation of $A \beta_{42}$ low-n oligomerization using a novel yeast reporter system. BMC Biol 4:32

Bassett DE Jr, Boguski MS, Hieter P (1996) Yeast genes and human disease. Nature 379:589-590

Bonawitz ND, Chatenay-Lapointe M, Pan Y, Shadel GS (2007) Reduced TOR signaling extends chronological life span via increased respiration and upregulation of mitochondrial gene expression. Cell Metab 5:265-277

Budovskaya YV, Stephan JS, Reggiori F, Klionsky DJ, Herman PK (2004) The Ras/cAMPdependent protein kinase signaling pathway regulates an early step of the autophagy process in Saccharomyces cerevisiae. J Biol Chem 279:20663-20671

Buttner S et al (2008) Functional mitochondria are required for alpha-synuclein toxicity in aging yeast. J Biol Chem 283:7554-7560

Chen Q, Thorpe J, Dohmen JR, Li F, Keller JN (2006) Ump1 extends yeast lifespan and enhances viability during oxidative stress: central role for the proteasome? Free Radic Biol Med 40:120-126

Chen Q, Thorpe J, Keller JN (2005) Alpha-synuclein alters proteasome function, protein synthesis, and stationary phase viability. J Biol Chem 280:30009-30017

Choubey V et al (2011) Mutant A53T alpha-synuclein induces neuronal death by increasing mitochondrial autophagy. J Biol Chem 286:10814-10824

Cooper AA et al (2006) Alpha-synuclein blocks ER-Golgi traffic and Rab1 rescues neuron loss in Parkinson's models. Science 313:324-328

Corcoles-Saez I, Dong K, Johnson AL, Waskiewicz E, Costanzo M, Boone C, Cha RS (2018) Essential function of Mec1, the budding yeast ATM/ATR checkpoint-response kinase protein homeostasis. Dev Cell 46(495-503):e492 
D’Angelo F, Vignaud H, Di Martino J, Salin B, Devin A, Cullin C, Marchal C (2013) A yeast model for amyloid-beta aggregation exemplifies the role of membrane trafficking and PICALM in cytotoxicity. Dis Model Mech 6:206-216

da Cunha FM, Demasi M, Kowaltowski AJ (2011) Aging and calorie restriction modulate yeast redox state, oxidized protein removal, and the ubiquitin-proteasome system. Free Radic Biol Med 51:664-670

Dang W et al (2009) Histone H4 lysine 16 acetylation regulates cellular lifespan. Nature 459:802-807

Delaney JR et al (2013) Dietary restriction and mitochondrial function link replicative and chronological aging in Saccharomyces cerevisiae. Exp Gerontol 48:1006-1013

Denoth Lippuner A, Julou T, Barral Y (2014) Budding yeast as a model organism to study the effects of age. FEMS Microbiol Rev 38:300-325

Deprez MA, Eskes E, Wilms T, Ludovico P, Winderickx J (2018) pH homeostasis links the nutrient sensing PKA/TORC1/Sch9 menage-a-trois to stress tolerance and longevity. Microb Cell 5:119-136

DiLoreto R, Murphy CT (2015) The cell biology of aging. Mol Biol Cell 26:4524-4531

Duina AA, Kalton HM, Gaber RF (1998) Requirement for Hsp90 and a CyP-40-type cyclophilin in negative regulation of the heat shock response. J Biol Chem 273:18974-18978

Dyavaiah M, Rooney JP, Chittur SV, Lin Q, Begley TJ (2011) Autophagy-dependent regulation of the DNA damage response protein ribonucleotide reductase 1. Mol Cancer Res 9:462-475

Eapen VV et al (2017) A pathway of targeted autophagy is induced by DNA damage in budding yeast. Proc Natl Acad Sci USA 114:E1158-E1167

Eisenberg T et al (2009) Induction of autophagy by spermidine promotes longevity. Nat Cell Biol 11:1305-1314

Eisenberg $\mathrm{T}$ et al (2014) Nucleocytosolic depletion of the energy metabolite acetyl-coenzyme a stimulates autophagy and prolongs lifespan. Cell Metab 19:431-444

Erjavec N, Larsson L, Grantham J, Nystrom T (2007) Accelerated aging and failure to segregate damaged proteins in Sir2 mutants can be suppressed by overproducing the protein aggregationremodeling factor Hsp104p. Genes Dev 21:2410-2421

Erjavec N, Nystrom T (2007) Sir2p-dependent protein segregation gives rise to a superior reactive oxygen species management in the progeny of Saccharomyces cerevisiae. Proc Natl Acad Sci USA 104:10877-10881

Escusa-Toret S, Vonk WI, Frydman J (2013) Spatial sequestration of misfolded proteins by a dynamic chaperone pathway enhances cellular fitness during stress. Nat Cell Biol 15:1231-1243

Ewald JC, Kuehne A, Zamboni N, Skotheim JM (2016) The yeast cyclin-dependent kinase routes carbon fluxes to fuel cell cycle progression. Mol Cell 62:532-545

Fabrizio P, Gattazzo C, Battistella L, Wei M, Cheng C, McGrew K, Longo VD (2005) Sir2 blocks extreme life-span extension. Cell 123:655-667

Fabrizio P et al (2010) Genome-wide screen in Saccharomyces cerevisiae identifies vacuolar protein sorting, autophagy, biosynthetic, and tRNA methylation genes involved in life span regulation. PLoS Genet 6:e1001024

Fabrizio P, Longo VD (2003) The chronological life span of Saccharomyces cerevisiae. Aging Cell 2:73-81

Finkel T, Deng CX, Mostoslavsky R (2009) Recent progress in the biology and physiology of sirtuins. Nature 460:587-591

Fontana L, Partridge L, Longo VD (2010) Extending healthy life span-from yeast to humans. Science 328:321-326

Galluzzi L et al (2017) Molecular definitions of autophagy and related processes. EMBO J 36:1811-1836

Garay E, Campos SE, Gonzalez de la Cruz J, Gaspar AP, Jinich A, Deluna A (2014) High-resolution profiling of stationary-phase survival reveals yeast longevity factors and their genetic interactions. PLoS Genet 10:e1004168 
Ghavidel A et al (2015) A genome scale screen for mutants with delayed exit from mitosis: Ire1independent induction of autophagy integrates ER homeostasis into mitotic lifespan. PLoS Genet 11:e1005429

Guedes A, Ludovico P, Sampaio-Marques B (2017) Caloric restriction alleviates alpha-synuclein toxicity in aged yeast cells by controlling the opposite roles of Tor1 and Sir2 on autophagy. Mech Ageing Dev 161:270-276

Harris N, MacLean M, Hatzianthis K, Panaretou B, Piper PW (2001) Increasing Saccharomyces cerevisiae stress resistance, through the overactivation of the heat shock response resulting from defects in the Hsp90 chaperone, does not extend replicative life span but can be associated with slower chronological ageing of nondividing cells. Mol Genet Genomics MGG 265:258-263

Higuchi-Sanabria R, Pernice WM, Vevea JD, Alessi Wolken DM, Boldogh IR, Pon LA (2014) Role of asymmetric cell division in lifespan control in Saccharomyces cerevisiae. FEMS Yeast Res 14:1133-1146

Hill SM, Hanzen S, Nystrom T (2017) Restricted access: spatial sequestration of damaged proteins during stress and aging. EMBO Rep 18:377-391

Howitz KT et al (2003) Small molecule activators of sirtuins extend Saccharomyces cerevisiae lifespan. Nature 425:191-196

Hughes AL, Gottschling DE (2012) An early age increase in vacuolar pH limits mitochondrial function and lifespan in yeast. Nature 492:261-265

Jiang JC, Jaruga E, Repnevskaya MV, Jazwinski SM (2000) An intervention resembling caloric restriction prolongs life span and retards aging in yeast. FASEB J 14:2135-2137

Kaeberlein M (2010) Lessons on longevity from budding yeast. Nature 464:513-519

Kaeberlein M, Burtner CR, Kennedy BK (2007) Recent developments in yeast aging. PLoS Genet 3:e84

Kaeberlein M, Kirkland KT, Fields S, Kennedy BK (2004) Sir2-independent life span extension by calorie restriction in yeast. PLoS Biol 2:E296

Kaeberlein M, McVey M, Guarente L (1999) The SIR2/3/4 complex and SIR2 alone promote longevity in Saccharomyces cerevisiae by two different mechanisms. Genes Dev 13:2570-2580

Kaeberlein M, Powers RW 3rd (2007) Sir2 and calorie restriction in yeast: a skeptical perspective. Ageing Res Rev 6:128-140

Kaeberlein M et al (2005) Regulation of yeast replicative life span by TOR and Sch9 in response to nutrients. Science 310:1193-1196

Kaganovich D, Kopito R, Frydman J (2008) Misfolded proteins partition between two distinct quality control compartments. Nature 454:1088-1095

Kirchman PA, Kim S, Lai CY, Jazwinski SM (1999) Interorganelle signaling is a determinant of longevity in Saccharomyces cerevisiae. Genetics 152:179-190

Kruegel U et al (2011) Elevated proteasome capacity extends replicative lifespan in Saccharomyces cerevisiae. PLoS Genet 7:e1002253

Kumar PA, Kumar MS, Reddy GB (2007) Effect of glycation on alpha-crystallin structure and chaperone-like function. Biochem J 408:251-258

Labbadia J, Morimoto RI (2014) Proteostasis and longevity: when does aging really begin? F1000prime Reports 6:7

Lam YT, Aung-Htut MT, Lim YL, Yang H, Dawes IW (2011) Changes in reactive oxygen species begin early during replicative aging of Saccharomyces cerevisiae cells. Free Radic Biol Med 50:963-970

Lavoie H, Whiteway M (2008) Increased respiration in the sch9Delta mutant is required for increasing chronological life span but not replicative life span. Eukaryot Cell 7:1127-1135

Lee HY, Chao JC, Cheng KY, Leu JY (2018) Misfolding-prone proteins are reversibly sequestered to an Hsp42-associated granule upon chronological aging. J Cell Sci 131

Lee HY, Cheng KY, Chao JC, Leu JY (2016) Differentiated cytoplasmic granule formation in quiescent and non-quiescent cells upon chronological aging. Microb Cell 3:109-119 
Leonov A et al (2017) Caloric restriction extends yeast chronological lifespan via a mechanism linking cellular aging to cell cycle regulation, maintenance of a quiescent state, entry into a non-quiescent state and survival in the non-quiescent state. Oncotarget 8:69328-69350

Li L, Miles S, Melville Z, Prasad A, Bradley G, Breeden LL (2013) Key events during the transition from rapid growth to quiescence in budding yeast require posttranscriptional regulators. Mol Biol Cell 24:3697-3709

Lin SJ, Defossez PA, Guarente L (2000) Requirement of NAD and SIR2 for life-span extension by calorie restriction in Saccharomyces cerevisiae. Science 289:2126-2128

Lindstrom DL, Leverich CK, Henderson KA, Gottschling DE (2011) Replicative age induces mitotic recombination in the ribosomal RNA gene cluster of Saccharomyces cerevisiae. PLoS Genet 7:e1002015

Liu B, Larsson L, Caballero A, Hao X, Oling D, Grantham J, Nystrom T (2010) The polarisome is required for segregation and retrograde transport of protein aggregates. Cell 140:257-267

Longo VD, Shadel GS, Kaeberlein M, Kennedy B (2012) Replicative and chronological aging in Saccharomyces cerevisiae. Cell Metab 16:18-31

Lopez-Otin C, Blasco MA, Partridge L, Serrano M, Kroemer G (2013) The hallmarks of aging. Cell 153:1194-1217

Lu JY et al (2011) Acetylation of yeast AMPK controls intrinsic aging independently of caloric restriction. Cell 146:969-979

Ludovico P, Burhans WC (2014) Reactive oxygen species, ageing and the hormesis police. FEMS Yeast Res 14:33-39

Ma Y, Li J (2015) Metabolic shifts during aging and pathology. Comprehensive Physiology 5:667-686

Matecic M, Smith DL, Pan X, Maqani N, Bekiranov S, Boeke JD, Smith JS (2010) A microarraybased genetic screen for yeast chronological aging factors. PLoS Genet 6:e1000921

McCormick MA et al (2015) A comprehensive analysis of replicative lifespan in 4,698 single-gene deletion strains uncovers conserved mechanisms of aging. Cell Metab 22:895-906

McFaline-Figueroa JR et al (2011) Mitochondrial quality control during inheritance is associated with lifespan and mother-daughter age asymmetry in budding yeast. Aging Cell 10:885-895

Medicherla B, Goldberg AL (2008) Heat shock and oxygen radicals stimulate ubiquitin-dependent degradation mainly of newly synthesized proteins. J Cell Biol 182:663-673

Meijer AJ, Codogno P (2007) Macroautophagy: protector in the diabetes drama? Autophagy 3:523-526

Mesquita A et al (2010) Caloric restriction or catalase inactivation extends yeast chronological lifespan by inducing $\mathrm{H}_{2} \mathrm{O}_{2}$ and superoxide dismutase activity. Proc Natl Acad Sci USA 107:15123-15128

Miles S, Li L, Davison J, Breeden LL (2013) Xbp1 directs global repression of budding yeast transcription during the transition to quiescence and is important for the longevity and reversibility of the quiescent state. PLoS Genet 9:e1003854

Miller SB et al (2015) Compartment-specific aggregases direct distinct nuclear and cytoplasmic aggregate deposition. EMBO J 34:778-797

Miller-Fleming L, Giorgini F, Outeiro TF (2008) Yeast as a model for studying human neurodegenerative disorders. Biotechnol J 3:325-338

Morimoto RI, Cuervo AM (2014) Proteostasis and the aging proteome in health and disease. J Gerontol Ser A Biol Sci Med Sci 69(Suppl 1):S38-S33

Morselli E et al (2011) Spermidine and resveratrol induce autophagy by distinct pathways converging on the acetylproteome. J Cell Biol 192:615-629

Mortimer RK, Johnston JR (1959) Life span of individual yeast cells. Nature 183:1751-1752

Muller I, Zimmermann M, Becker D, Flomer M (1980) Calendar life span versus budding life span of Saccharomyces cerevisiae. Mech Ageing Dev 12:47-52

Murakami C et al (2012) pH neutralization protects against reduction in replicative lifespan following chronological aging in yeast. Cell Cycle 11:3087-3096 
Nakamura N, Matsuura A, Wada Y, Ohsumi Y (1997) Acidification of vacuoles is required for autophagic degradation in the yeast, Saccharomyces cerevisiae. J Biochem 121:338-344

Noda T, Ohsumi Y (1998) Tor, a phosphatidylinositol kinase homologue, controls autophagy in yeast. J Biol Chem 273:3963-3966

Ocampo A, Liu J, Schroeder EA, Shadel GS, Barrientos A (2012) Mitochondrial respiratory thresholds regulate yeast chronological life span and its extension by caloric restriction. Cell Metab 16:55-67

Oliveira AV, Vilaca R, Santos CN, Costa V, Menezes R (2017) Exploring the power of yeast to model aging and age-related neurodegenerative disorders. Biogerontology 18:3-34

Outeiro TF, Lindquist S (2003) Yeast cells provide insight into alpha-synuclein biology and pathobiology. Science 302:1772-1775

Pan Y, Schroeder EA, Ocampo A, Barrientos A, Shadel GS (2011) Regulation of yeast chronological life span by TORC1 via adaptive mitochondrial ROS signaling. Cell Metab 13:668-678

Park SK, Pegan SD, Mesecar AD, Jungbauer LM, LaDu MJ, Liebman SW (2011) Development and validation of a yeast high-throughput screen for inhibitors of $A \beta_{42}$ oligomerization. Dis Model Mech 4:822-831

Peric M et al (2016) Crosstalk between cellular compartments protects against proteotoxicity and extends lifespan. Sci Rep 6:28751

Petroi D et al (2012) Aggregate clearance of alpha-synuclein in Saccharomyces cerevisiae depends more on autophagosome and vacuole function than on the proteasome. J Biol Chem 287:27567-27579

Piper PW (2006) Long-lived yeast as a model for ageing research. Yeast 23:215-226

Piper PW, Harris NL, MacLean M (2006) Preadaptation to efficient respiratory maintenance is essential both for maximal longevity and the retention of replicative potential in chronologically ageing yeast. Mech Ageing Dev 127:733-740

Reggiori F, Klionsky DJ (2013) Autophagic processes in yeast: mechanism, machinery and regulation. Genetics 194:341-361

Ritz P, Berrut G (2005) Mitochondrial function, energy expenditure, aging and insulin resistance. Diabetes Metab 31(Spec No 2):5S67-65S73

Rockenfeller P et al (2015) Phosphatidylethanolamine positively regulates autophagy and longevity. Cell Death Differ 22:499-508

Rogina B, Helfand SL (2004) Sir2 mediates longevity in the fly through a pathway related to calorie restriction. Proc Natl Acad Sci USA 101:15998-16003

Rubinsztein DC, Marino G, Kroemer G (2011) Autophagy and aging. Cell 146:682-695

Ruckenstuhl C et al (2014) Lifespan extension by methionine restriction requires autophagydependent vacuolar acidification. PLoS Genet 10:e1004347

Ruetenik A, Barrientos A (2015) Dietary restriction, mitochondrial function and aging: from yeast to humans. Biochem Biophys Acta 1847:1434-1447

Sampaio-Marques B, Burhans WC, Ludovico P (2014a) Longevity pathways and maintenance of the proteome: the role of autophagy and mitophagy during yeast ageing. Microb Cell 1:118-127

Sampaio-Marques B, Burhans WC, Ludovico P (2014b) Longevity pathways and maintenance of the proteome: the role of autophagy and mitophagy during yeast ageing. Microbial Cell 1:118-127

Sampaio-Marques B et al (2012) SNCA (alpha-synuclein)-induced toxicity in yeast cells is dependent on sirtuin 2 (Sir2)-mediated mitophagy. Autophagy 8:1494-1509

Sampaio-Marques B, Ludovico P (2015) Sirtuins and proteolytic systems: implications for pathogenesis of synucleinopathies. Biomolecules 5:735-757

Sampaio-Marques B, Ludovico P (2018) Linking cellular proteostasis to yeast longevity. FEMS Yeast Res 18

Schroeder EA, Raimundo N, Shadel GS (2013) Epigenetic silencing mediates mitochondria stressinduced longevity. Cell Metab 17:954-964

Seynnaeve D et al (2018) Recent insights on Alzheimer's disease originating from yeast models. Int J Mol Sci 19 
Sharma N, Brandis KA, Herrera SK, Johnson BE, Vaidya T, Shrestha R, Debburman SK (2006) Alpha-synuclein budding yeast model: toxicity enhanced by impaired proteasome and oxidative stress. J Mol Neurosci 28:161-178

Sinclair DA, Guarente L (1997) Extrachromosomal rDNA circles-a cause of aging in yeast. Cell 91:1033-1042

Smets B, Ghillebert R, De Snijder P, Binda M, Swinnen E, De Virgilio C, Winderickx J (2010) Life in the midst of scarcity: adaptations to nutrient availability in Saccharomyces cerevisiae. Curr Genet 56:1-32

Smith DL Jr, McClure JM, Matecic M, Smith JS (2007) Calorie restriction extends the chronological lifespan of Saccharomyces cerevisiae independently of the Sirtuins. Aging Cell 6:649-662

Smith J, Schneider BL (2018) A budding topic: modeling aging and longevity in yeast. In: Conn's handbook of models for human aging, pp 389-415

Stefanis L, Larsen KE, Rideout HJ, Sulzer D, Greene LA (2001) Expression of A53T mutant but not wild-type alpha-synuclein in PC12 cells induces alterations of the ubiquitin-dependent degradation system, loss of dopamine release, and autophagic cell death. J Neurosci 21:9549-9560

Tenreiro S, Franssens V, Winderickx J, Outeiro TF (2017) Yeast models of Parkinson's diseaseassociated molecular pathologies. Curr Opin Genet Dev 44:74-83

Tenreiro S, Munder MC, Alberti S, Outeiro TF (2013) Harnessing the power of yeast to unravel the molecular basis of neurodegeneration. J Neurochem 127:438-452

Tenreiro S, Outeiro TF (2010) Simple is good: yeast models of neurodegeneration. FEMS Yeast Res 10:970-979

Tissenbaum HA, Guarente L (2001) Increased dosage of a sir-2 gene extends lifespan in Caenorhabditis elegans. Nature 410:227-230

Treusch S et al (2011) Functional links between Abeta toxicity, endocytic trafficking, and Alzheimer's disease risk factors in yeast. Science 334:1241-1245

Tyler JK, Johnson JE (2018a) The role of autophagy in the regulation of yeast life span. Ann N Y Acad Sci 1418:31-43

Tyler JK, Johnson JE (2018b) The role of autophagy in the regulation of yeast life span. Ann N Y Acad Sci

Vandebroek T et al (2005) Identification and isolation of a hyperphosphorylated, conformationally changed intermediate of human protein tau expressed in yeast. Biochemistry 44:11466-11475

Vanhelmont $T$ et al (2010) Serine-409 phosphorylation and oxidative damage define aggregation of human protein tau in yeast. FEMS Yeast Res 10:992-1005

Vanhooren V et al (2015) Protein modification and maintenance systems as biomarkers of ageing. Mech Ageing Dev 151:71-84

Verduyckt M, Vignaud H, Bynens T, Van den Brande J, Franssens V, Cullin C, Winderickx J (2016) Yeast as a model for Alzheimer's disease: latest studies and advanced strategies. Methods Mol Biol 1303:197-215

Vilaca $R$ et al (2018) The ceramide activated protein phosphatase Sit4 impairs sphingolipid dynamics, mitochondrial function and lifespan in a yeast model of Niemann-Pick type C1. Biochim Biophys Acta Mol Basis Dis 1864:79-88

Wang Z, Wilson WA, Fujino MA, Roach PJ (2001) Antagonistic controls of autophagy and glycogen accumulation by Snflp, the yeast homolog of AMP-activated protein kinase, and the cyclindependent kinase Pho85p. Mol Cell Biol 21:5742-5752

Weinberger M, Sampaio-Marques B, Ludovico P, Burhans WC (2013) DNA replication stressinduced loss of reproductive capacity in S. cerevisiae and its inhibition by caloric restriction. Cell Cycle 12:1189-1200

Werner-Washburne M, Roy S, Davidson GS (2012) Aging and the survival of quiescent and nonquiescent cells in yeast stationary-phase cultures Subcell. Biochem 57:123-143

Wierman MB, Smith JS (2014) Yeast sirtuins and the regulation of aging. FEMS Yeast Res 14:73-88

Xilouri M, Vogiatzi T, Vekrellis K, Park D, Stefanis L (2009) Abberant alpha-synuclein confers toxicity to neurons in part through inhibition of chaperone-mediated autophagy. PLoS ONE 4:e5515 
Yi C et al (2012) Function and molecular mechanism of acetylation in autophagy regulation. Science 336:474-477

Yi DG, Hong S, Huh WK (2018) Mitochondrial dysfunction reduces yeast replicative lifespan by elevating RAS-dependent ROS production by the ER-localized NADPH oxidase Yno1. PLoS One 13:e0198619

Yin Z, Pascual C, Klionsky DJ (2016) Autophagy: machinery and regulation. Microb Cell 3:588-596

Yorimitsu T, Zaman S, Broach JR, Klionsky DJ (2007) Protein kinase A and Sch9 cooperatively regulate induction of autophagy in Saccharomyces cerevisiae. Mol Biol Cell 18:4180-4189

Zhou C et al (2014) Organelle-based aggregation and retention of damaged proteins in asymmetrically dividing cells. Cell 159:530-542 